\title{
THE LENGTH OF A CURVE IN A SPACE OF CURVATURE $<K$
}

\author{
B. V. DEKSTER ${ }^{1}$
}

\begin{abstract}
Let $M$ be a compact ball in a Riemannian manifold with sectional curvatures $<K$. Suppose its radius $R_{0}$ is less than the injectivity radius at the center of $M$ and $R_{0}<\pi / 2 \sqrt{K}$ if $K>0$. Denote by $M_{0}$ a circle of radius $R_{0}$ in the plane of constant curvature $K$ and by $\kappa$ the curvature of $\partial M_{0}$. Then any curve in $M$ with curvature $<\chi<\kappa$ is not longer than a circular arc of curvature $\chi$ in $M_{0}$ whose ends are opposite points of $\partial M_{0}$. Any curve in $M$ with total curvature not exceeding some $\tau>0\left(\tau=\pi / 2\right.$ if $\left.K<\kappa^{2}\right)$ is not longer than the longest curve in $M_{0}$ with the same total curvature whose tangent vector rotates in a permanent direction.
\end{abstract}

\section{Introduction.}

0.1. We establish here (Theorems 1.2, 1.3, 1.6) upper bounds of the length of a curve in a (convex) ball in a Riemannian space with sectional curvatures (in the ball) $\leqslant K$. The upper bounds are expressed in terms of $K$, the radius of the ball and curvature of the curve.

Some applications of these estimates are brought out in \$2. Say any infinitely long curve in a simply connected complete space of curvature $<K<0$ goes to $\infty$ if its total curvature does not grow "too fast" (or just finite), see 2.7. We establish in 2.8 how fast the curve goes to $\infty$ in terms of its curvature.

0.2. Results of a similar nature are proved in [2] and [3]. The difference is that there the ball is replaced by a more general convex region, instead of $K$ a lower bound $k_{s}$ of sectional curvatures in the region is used and the role of the radius is played by the minimal normal curvature $\kappa$ of the boundary of the region. Moreover, the condition $k_{s}>-\kappa^{2}$ is supposed to hold in [2], [3].

We apply here a modification of the method used in [3] (rolling a curve along a fixed one, see [3, 3.11]).

0.3. As for the history of the problem, the length of a curve in a 2-dimensional surface was estimated by A. D. Aleksandrov and V. V. Strel'cov [1] in 1953. Their estimates and ours (when the dimension $n=2$ ) do not follow from one another.

In 1969, Gromoll and Meyer [4, Lemma 6] proved that for any compact set in a complete open manifold of positive curvature there exists a number such that the length of any geodesic in the set is less than that number.

0.4. In Euclidean case, a similar result is represented by Rešetnyak Theorem $[5$, p. 262]. Its simplified version is as follows.

Received by the editors August 6, 1978.

AMS (MOS) subject classifications (1970). Primary 53C40.

${ }^{1}$ Supported by NRC of Canada Grant awarded to Professor H.S.M. Coxeter.

(c) 1980 American Mathematical Society 0002-9939/80/0000-0274/\$03.00 
REŠETNYAK THEOREM. Let $x:[0, L] \rightarrow R^{n}$ be a piecewise regular curve parametrized by its arc length. Put $\delta=\max \Varangle(\dot{x}(a), \dot{x}(b))$ where $\Varangle$ means angle and the maximum is taken over all regularity points $a, b \in[0, L]$. If all vectors $\dot{x}(s)$ (in regularity points) are directed into the same half-space and $\cos \delta>-1 /(n-1)$, then

$$
L \leqslant \frac{r \sqrt{n}}{\sqrt{1+(n-1) \cos \delta}}
$$

where $r$ is the distance between $x(0)$ and $x(L)$.

\section{Curves in a ball.}

1.1. Basic notation and assumptions. All manifolds and curves here are supposed to be of class $C^{\infty}$ unless otherwise stated. A curve parametrized by arc length will be called normal. An oriented 2-dimensional sphere, plane or hyperbolic plane of curvature $K$ will be denoted by $P^{2}$.

For a normal curve $c:[0, L] \rightarrow P^{2}$, along with the ordinary curvature $|\ddot{c}|$, we consider oriented curvature, i.e., $|\ddot{c}|$ with ascribed sign $+(-)$ if $\dot{c}$ rotates in the positive (negative) direction. Total curvature of a curve and its total oriented curvature (if it lies in $P^{2}$ ) are integrals of the appropriate curvatures along the curve. These definitions are naturally generalized for a piecewise $C^{2}$-curve.

A minimal geodesic with the ends $a, b$ (in any space considered) is denoted sometimes by $a b$, its length by $\overline{a b}$ and its direction (unit vector at a point in $a b$ being under discussion) by $\overrightarrow{a b}$. The notations $\Varangle(\cdot, \cdot)$ and $\Varangle \ldots$ mean angle.

Throughout the paper, we denote by $M$ a compact ball in a Riemannian manifold and set $\Gamma=\partial M$. Let $K$ be an upper bound of sectional curvatures in $M$ and let $R_{0}$ be its radius. We suppose $R_{0}$ to be less than injectivity radius at the center of $M$ and

$$
R_{0}<\frac{\pi}{2 \sqrt{K}} \quad \text { if } K>0 .
$$

We assign to $M$ a circle $M_{0} \subset P^{2}$ of radius $R_{0}$ and denote by $\kappa=\kappa\left(R_{0}, K\right)$ the curvature of its circumference $\Gamma_{0}$. By (1.1), the circle $M_{0}$ is convex and $\kappa>0$. It follows easily from the Rauch Comparison Theorem that $M$ is convex as well.

The distance in $M$ is denoted by $\rho(\cdot, \cdot)$ and in $P^{2}$ by $\rho_{0}(\cdot, \cdot)$.

1.2. The following three theorems coincide literally with Theorems $1.5,1.6,1.10$ in [3] after replacement of $k_{s}$ there by $K$ (although $M$ and $M_{0}$ here have another meaning).

THEOREM. Any curve in $M$ with curvature at every point not greater than $\chi \in[0, \kappa)$ is not longer than a circular arc in $M_{0}$ of curvature $\chi$ whose ends are opposite points of $\Gamma_{0}$. (In particular, any geodesic in $M$ is not longer than $2 R_{0}$.)

1.3. Theorem. Let a curve of length $L$ lie in $M$ and have the total curvature $\theta$ satisfying

$$
\theta \in \begin{cases}{\left[0, \theta^{*}\right)} & \text { if } K>\kappa^{2} \\ {[0, \pi / 2]} & \text { otherwise }\end{cases}
$$


where the precise value of $\theta^{*}=\theta^{*}(\kappa, K)$ is given in 1.4. Denote by $l_{\theta}$ supremum of the lengths of piecewise $C^{2}$-curves in $M_{0}$ such that the straight line of support of each curve rotates in the same direction when moving along the curve and the total curvature of each curve does not exceed $\theta$. Then $l_{\theta}$ is finite and

$$
L<l_{\theta} \text {. }
$$

1.4. The condition $K>\kappa^{2}\left(=K \cot ^{2} \sqrt{K} R_{0}\right)$ in (1.1) implies $2 R_{0}>\pi / 2 \sqrt{K}$. Let $Y_{0} Z_{0}$ be a diameter of $M_{0}$ and $Z_{0} V_{0}$ be a chord of length $\pi / 2 \sqrt{K}$. Put $\delta=\Varangle Y_{0} Z_{0} V_{0}$ and denote by $\sigma$ the area of the part of $M_{0}$ bounded by $Y_{0} Z_{0}, Z_{0} V_{0}$

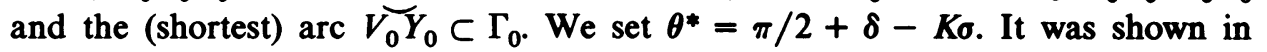
$[3,2.4]$ that $\theta^{*} \in(0, \pi / 2)$.

1.5. Denote by $J_{\theta}$ the class of the curves in $M_{0}$ mentioned in 1.3. Proposition 4.2 in [3] (with $k_{s}$ replaced by $K$ ) establishes that there is a longest curve $\gamma_{\theta}$ in the class $J_{\theta}$. It gives also the exact description of $\gamma_{\theta}$. The description shows that the length $l_{\theta}$ of $\gamma_{\theta}$ increases by $\theta$. In the case $K<\kappa^{2}, \gamma_{\theta}$ is a polygonal line $A C B$ with the ends $A, B$ in diametrically opposite points of $\Gamma_{0}, \overline{A C}=\overline{C B}$ and with the total curvature $\pi-\Varangle A C B=\theta$.

1.6. A nondecreasing piecewise $C^{1}$-function $\Xi:[0, \infty) \rightarrow R$ with $\Xi(0)=0$ will be called a turn-function if it is continuous from the left and any of its jumps is less than $\pi$.

Our main technical result is the following:

TheOREM. Let $d \in\left[0, R_{0}\right], \alpha \in[0, \pi]$ and let $\Xi$ be a turn-function. Denote by $M_{0}^{+}$ the closed semicircle separated from $M_{0}$ by a diameter $Y_{0} Z_{0}$ and such that rotation of the radius $0 Y_{0}$ to the radius $0 Z_{0}$ within $M_{0}^{+}$is positive. Suppose, there exists a mapping $\gamma_{0}$ such that

(i) $\gamma_{0}$ is a normal piecewise $C^{2}$-curve $[0, \infty) \rightarrow P^{2}$,

(ii) $\gamma_{0}(0) \in 0 Y_{0}$ and $\overline{Y_{0} \gamma_{0}(0)}=d$,

(iii) $\dot{\gamma}_{0}$ is directed into $M_{0}^{+}$and $\Varangle\left(\dot{\gamma}_{0}(0), \overrightarrow{0 Y_{0}}\right)=\alpha$,

(iv) the total oriented curvature of $\gamma_{0 \mid[0, s]}$ is equal to $\Xi(s), s>0$,

(v) there is a maximum number $L_{0}>0$ such that $\gamma_{0}\left(\left[0, L_{0}\right]\right) \subset M_{0}^{+}$,

(vi) $\gamma_{0}\left(L_{0}\right) \in \Gamma_{0} \cap M_{0}^{+} \backslash Z_{0}$ and the curve $\gamma_{0 \mid\left[0, L_{0}\right]}$, the segment $\gamma_{0}(0) Z_{0}$ and the arc $\gamma_{0}\left(\bar{L}_{0}\right) Z_{0}$ of the semicircle $\Gamma_{0} \cap M_{0}^{+}$bound a nondegenerate region. (This implies that the region is convex and $\alpha<\pi$.)

Then the length $L$ of any normal curve $\gamma:[0, L] \rightarrow M$ does not exceed $L_{0}$ if

(I) $\rho(\gamma(0), \Gamma)=d$,

(II) in the case $d \neq R_{0}, \dot{\gamma}(0)$ forms an angle $\phi<\alpha$ with the radius coming through $\gamma(0)$ and directed from the center of $M$,

(III) $|\ddot{\gamma}(t)| \leqslant \Xi^{\prime}(t)\left(=\left|\ddot{\gamma}_{0}(t)\right|\right)$ for those $t \in[0, L]$ where $\Xi^{\prime}$ exists.

\section{Some remarks and applications.}

2.1. Theorems 1.3 and 1.6 are naturally generalized for the case of a piecewise $C^{2}$-curve in $M$.

2.2. Let $\gamma:[0, L] \rightarrow M$ be a normal piecewise $C^{2}$-curve of total curvature $\theta>0$. Let $\theta=\sum_{i=1}^{m} \theta_{i}$ where each $\theta_{i}$ satisfies (1.2). Then $L<\sum_{i=1}^{m} l_{\theta_{i}}$ where $l_{\theta_{1}}$ is as in Theorem 1.3. 
Indeed, $\gamma$ can be divided into $m$ parts of total curvature $\tau_{i}<\theta_{i}, i=1,2, \ldots, m$. Applying Theorem 1.3 to each part and adding, we have

$$
L \leqslant \sum_{i=1}^{m} l_{\tau_{i}} \leqslant \sum_{i=1}^{m} l_{\theta_{i}}
$$

see 1.5.

2.3. The equalities hold in the estimates of $1.2,1.3$ and 1.6 when $M=M_{0}$ and the curve in $M$ mentioned there coincides with a circular arc of curvature $\chi$ having the ends in opposite points of $\Gamma_{0}$, with $\gamma_{\theta}$ (see 1.5) and $\gamma_{0 \mid\left[0, L_{0}\right]}$, respectively.

2.4. Let for example $M$ be a ball of radius $R_{0}$ in $R^{n}$ and $\theta=\pi / 2$. Take $K=0$. Then $M_{0}$ is a circle of radius $R_{0}$ in $R^{2}$ and we may imbed $M_{0}$ into $M$. According to $1.5, l_{\theta}=l_{\pi / 2}=2 \sqrt{2} R_{0}$. So, by Theorem 1.3, any curve with total curvature $<\pi / 2$ in the ball $M$ has the length $\leqslant 2 \sqrt{2} R_{0}$. This estimate is realized by a polygonal line $A C B \subset M_{0} \subset M$ with $A, C, B \subset \partial M_{0} \subset \partial M, \overline{A C}=\overline{C B}$ and $\Varangle A C B=\pi / 2$.

The Rešetnyak Theorem 0.4 (which is also exact) applied to $A C B$ yields a rougher estimate:

$$
\overline{A C}+\overline{C B} \leqslant \frac{2 R_{0} \sqrt{n}}{\sqrt{1+(n-1) \cos \pi / 2}}=2 \sqrt{n} R_{0} .
$$

2.5. In the rest of $\S 2, N$ means a complete simply connected Riemannian space with sectional curvatures $\leqslant K \leqslant 0$. Let $\gamma:[0, \infty) \rightarrow N$ be a normal curve, $\xi(s)$ be its curvature and $\theta(s)=\int_{0}^{s} \xi(x) d x$. We denote by $B_{t}(p)$ the closed ball of radius $t$ centered at $p \in N$. By the Hadamard-Cartan Theorem, it is homeomorphic to a ball.

2.6. Let us show that $\gamma$ goes to $\infty$ if $\overline{\lim }_{s \rightarrow \infty} \xi(s)<\sqrt{-K}$. Notice that $\kappa\left(R_{0}, K\right)$ (see 1.1) decreases by $R_{0}$ and $\lim _{R_{0} \rightarrow \infty} \kappa\left(R_{0}, K\right)=\sqrt{-K}$. Suppose now the contrary, i.e., that $\gamma$ can be included in a ball $B_{t}(p)$. The curvature $\kappa(t, K)$ of the boundary of the circle $M_{0}$ (constructed for the ball $M=B_{t}(p)$ ) is greater than $\sqrt{-K}$. Then $\xi(s)<\kappa(t, K)$ for $s$ larger than some $s_{0}$. By Theorem 1.2, any piece of $\gamma_{\left.\| s_{0, \infty} \infty\right)}$ is not longer than the arc mentioned there which is impossible. (The remark 2.6 can be proved without reference to Theorem 1.2 and probably is known.)

2.7. In 2.7-2.8, we take $K=0$. Let us represent $\theta(s)$ as $(m-1)(\pi / 2)+\tilde{\theta}$ where $m$ is an integer and $\tilde{\theta} \in[0, \pi / 2)$. If $\gamma_{[[0, s]}$ lies in a ball $B_{t}(p)$ then, by 2.2 ,

$$
s \leqslant(m-1) l_{\pi / 2}+l_{\tilde{\theta}}<m l_{\pi / 2} \leqslant\left(\frac{\theta(s)}{\pi / 2}+1\right) \cdot 2 \sqrt{2} t .
$$

We can see now that $\gamma$ goes to $\infty$ if $\lim _{s \rightarrow \infty} \theta(s) / s=0$. Indeed, otherwise $\gamma([0, \infty)) \subset B_{t}(p)$ and $(2.1)$ holds for any $s>0$ so that

$$
\lim _{s \rightarrow \infty} \frac{\theta(s)}{s} \geqslant \lim _{s \rightarrow \infty}\left(\frac{1}{2 \sqrt{2} t}-\frac{1}{s}\right) \frac{\pi}{2}=\frac{\pi}{4 \sqrt{2} t}>0 .
$$

2.8. Suppose there are numbers $A>0, \alpha \in[0,1)$ such that $\theta(s)<A s^{\alpha}$ for sufficiently large $s$. By $2.7, \gamma$ goes to $\infty$. Take an arbitrary point $p \in N$. For $t>\overline{p \gamma(0)}$, denote by $s(t)$ the maximum number such that $\gamma([0, s(t)]) \subset B_{t}(p)$. Then 


$$
\varlimsup_{t \rightarrow \infty} \frac{s(t)}{t^{1 /(1-\alpha)}} \leqslant \begin{cases}\left(\frac{2 \sqrt{2} A}{\pi / 2}\right)^{1 /(1-\alpha)} & \text { if } \alpha \in(0,1), \\ 2 \sqrt{2}\left(\frac{A}{\pi / 2}+1\right) & \text { if } \alpha=0\end{cases}
$$

Proof. By (2.1),

$$
s(t)<\left(\frac{A s^{\alpha}(t)}{\pi / 2}+1\right) 2 \sqrt{2} t .
$$

Suppose first $\alpha \neq 0$. Fix some $\varepsilon>0$. When $t$ (and $s(t)$ ) are sufficiently large then $\left(A s^{\alpha}(t) /(\pi / 2)\right) \varepsilon>1$. Now (2.3) implies

$$
\begin{aligned}
& s(t) \leqslant \frac{A s^{\alpha}(t)}{\pi / 2}(1+\varepsilon) 2 \sqrt{2} t, \\
& s(t) \leqslant\left[(1+\varepsilon) \frac{2 \sqrt{2} A}{\pi / 2} t\right]^{1 /(1-\alpha)} .
\end{aligned}
$$

Dividing this by $t^{1 /(1-\alpha)}$, passing to the upper limit as $t \rightarrow \infty$ and then to the limit as $\varepsilon \rightarrow 0$, one gets $(2.2)$ for $\alpha \in(0,1)$.

For $\alpha=0,(2.2)$ is proved by dividing (2.3) by $t$ and passing to the upper limit.

Notice that the case $\alpha>1$ and the case $\alpha=1$ with sufficiently large $A$ are not of interest since then in $N$ there always exists a curve $\gamma$ (any periodic curve) satisfying $\theta(s)<A s^{\alpha}$ but not going to $\infty$.

\section{Proofs of Theorems of $\$ 1$.}

3.1. Proof of Theorem 1.2. Let $b:[0, L] \rightarrow M$ be a normal curve with $|\ddot{b}|<\chi<\kappa$. Let $b(\lambda), \lambda \in[0, L]$, be a point closest to the center $C$ of $M$. Put $\gamma(t)=b(\lambda+t), t$ $\in[0, L-\lambda]$. Let the radius $C Y \ni \gamma(0)$. Obviously, $\Varangle(\dot{\gamma}(0), \overrightarrow{C Y})=\pi / 2$. (If $\gamma(0)$ $=C$, we choose a radius with this property.) Since $\chi<\kappa$, there exists the circumference $\gamma_{0}$ of curvature $\chi$ satisfying the conditions (i)-(vi) of Theorem 1.6 for the set $d=\overline{Y_{\gamma}(0)}, \alpha=\pi / 2, \Xi(t)=\chi t$. Since $|\ddot{\gamma}(t)|=|\ddot{b}(\lambda+t)|<\chi=\Xi^{\prime}$, the conditions (I)-(III) hold as well. Then $L-\lambda<L_{0}$ (see $1.6(\mathrm{v})$ ).

Similarly, $\lambda<L_{0}$. Addition yields $L<2 L_{0}$. But $2 L_{0}$ is the length of a circular arc of curvature $\chi$ in $M_{0}$ consisting of $\gamma_{0}\left(\left[0, L_{0}\right]\right)$ and its specular reflection with respect to the diameter $Y_{0} Z_{0}$. Obviously, this arc is not longer than the one mentioned in Theorem 1.2.

3.2. Proof of Theorem 1.3. It coincides literally with Proof 2.5 (along with Lemma 2.2) in [3] if $k_{s}$ there is replaced by $K$ and the reference to Theorem 1.10 there is replaced by the reference to Theorem 1.6 of this paper. Moreover, since $M$ is a ball here, some obvious simplifications are available.

3.3. Proof of Theorem 1.6. It is close to the proof of Theorem 1.10 in $[3, \S 3]$ but much simpler and shorter since $M$ is a ball here. A principal difference will be remarked on in $\$ 3.6$.

By a simple limit reasoning, we may assume that $\Xi \in C^{1}$, (then $\gamma_{0} \in C^{2}$ ) and that $\gamma([0, L]) \subset$ int $M \backslash C$ where $C$ is the center of $M$. We assume also that there are no points where $\gamma$ is tangent to the radii of $M$. For the dimension $n>3$, such 
points can be easily eliminated by variation of $\gamma$. For $n=2$, a finite number of such points can be inevitable but they may be treated as in [3].

3.4. Denote by $p, \omega$ the polar coordinates in $P^{2}$ with the pole at the center 0 of $M_{0}$ and the angle $\omega$ counted in the positive direction from the radius $0 Y_{0}$. Put $r(t)=\rho(\gamma(t), \Gamma)$. By 3.3, $r(t) \in C^{\infty}, r(t) \in\left(0, R_{0}\right)$ and $\left|r^{\prime}\right|<1, t \in[0, L]$. Consider the curve $\gamma_{1}:[0, L] \rightarrow M_{0}$ with the equations

$$
\omega= \begin{cases}\sin \left(R_{0} \sqrt{K}\right) \int_{0}^{t} \frac{\sqrt{1-r^{\prime 2}(x)}}{\sin \left(R_{0}-r(x)\right) \sqrt{K}} d x & \text { if } K>0, \\ R_{0} \int_{0}^{t} \frac{\sqrt{1-r^{\prime 2}(x)}}{R_{0}-r(x)} d x & \text { if } K=0, \\ \sinh \left(R_{0} \sqrt{-K}\right) \int_{0}^{t} \frac{\sqrt{1-r^{\prime 2}(x)}}{\sinh \left(R_{0}-r(x)\right) \sqrt{-K}} d x & \text { if } K<0 .\end{cases}
$$

Obviously, $\gamma_{1} \in C^{\infty}$. One can check that $\left|\dot{\gamma}_{1}\right|=1$. So, $\gamma_{1}$ is a normal curve. Moreover, $\Varangle\left(\dot{\gamma}_{1}(0), \overrightarrow{0 Y_{0}}\right)=\cos ^{-1}-r^{\prime}(0)=\phi$ and $\gamma_{1}([0, L]) \in$ int $M_{0}$.

3.5. A simple calculation based on (3.1), (3.2) shows that the oriented curvature $\xi_{1}(t)$ of $\gamma_{1}$ satisfies

$$
r^{\prime \prime}=\sqrt{1-r^{\prime 2}} \xi_{1}-\left(1-r^{\prime 2}\right) \kappa_{r}
$$

where $\kappa_{r}(>0)$ is curvature of the circumference of radius $R_{0}-r$ centered at 0 . (It comes through $\gamma_{1}(t)$.) By (3.9) in [2],

$$
r^{\prime \prime}<\sqrt{1-r^{\prime 2}}|\ddot{\gamma}|-\left(1-r^{\prime 2}\right) x
$$

where $x$ is the normal curvature of the sphere (coming through $\gamma(t)$ ) of radius $\boldsymbol{R}_{\mathbf{0}}-\boldsymbol{r}$ centered at $\boldsymbol{C}$ on the side of the interior normal $\nu$ in the direction of $\dot{\gamma}-\nu\langle\dot{\gamma}, \nu\rangle(\neq 0$ since $|\langle\dot{\gamma}, \nu\rangle|\langle 1$, see 3.3).

It follows easily from Rauch Comparison Theorem that $x>\kappa_{r}$. Replacing $x$ by $\kappa_{r}$ in (3.4) and combining it with (3.3), one obtains $\xi_{1}<|\ddot{\gamma}|$. Along with the conditions (III) and (iv) of 1.6 , it yields

$$
\xi_{1}(t)<\Xi^{\prime}(t)=\xi_{0}(t), \quad t \in[0, L],
$$

where $\xi_{0}$ is the oriented curvature of $\gamma_{0}$.

3.6. The inequality (3.5) is what provides a desired property of rolling $\gamma_{0}$ along $\gamma_{1}$. It is the basis of the further geometric construction.

Notice that the inequality $x>\kappa_{r}$ from which (3.5) follows appeared in [2] and [3] as well but due to other reasons. In [2], the normal curvature $x$ (denoted there by $K)$ of the surface $\Gamma(r)$ parallel to $\Gamma$ and distant by $r$ from $\Gamma$ satisfies $x>\kappa_{r}$ for the following two reasons.

(1) By the construction, the curvature of $\Gamma_{0}$ there is a lower bound of normal curvatures of $\Gamma$.

(2) The curvature $\left(k_{s}\right)$ of $P^{2}$ there is a lower bound of sectional curvatures in $M$. 
Then (1) and (2) result in $x>\kappa_{r}$ by a version of the Rauch Comparison Theorem dealing with Jacobi fields associated with a hypersurface $(\Gamma)$. (See [2, (3.9), Lemma 4].)

3.7. To finish the proof, it is enough to show that the curve $\gamma_{0 \mid\left[0, L_{0}\right]}$ is not longer than $\gamma_{1}$. We describe here only the idea of the reasoning. (See $[3,83]$ for details.)

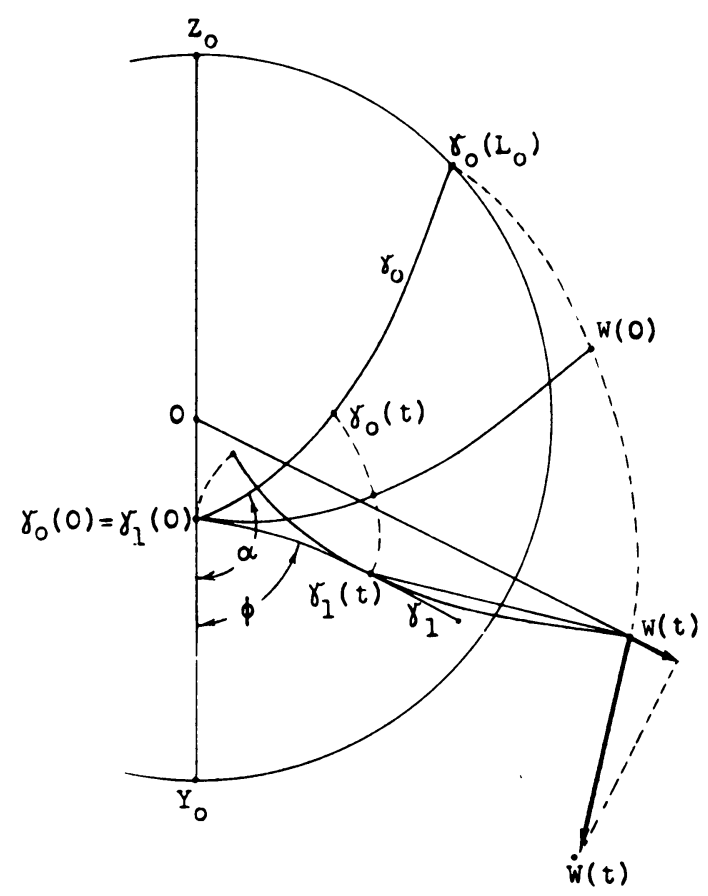

Let us rotate $\gamma_{0}$ about its end $\gamma_{0}(0)$ in the negative direction about the angle $\alpha-\phi>0$. Denote by $W(0)$ the new position of the point $\gamma_{0}\left(L_{0}\right)$, see the figure. Obviously, $W(0) \notin$ int $M_{0}$.

Now let $\gamma_{0}$ roll along $\gamma_{1}$ assuming that at a moment $t$ the new position of $\gamma_{0}(t)$ coincides with $\gamma_{1}(t)$. Instantaneously, $\gamma_{0}$ rotates about the point $\gamma_{1}(t)$ in the negative direction with the angular speed $\xi_{0}(t)-\xi_{1}(t)>0$, see (3.5). Let $W(t)$ be the position of $\gamma_{0}\left(L_{0}\right)$ at a moment $t$ and $\dot{W}(t)$ be its speed. Then $\dot{W}(t) \perp W(t) \gamma_{1}(t)$ and therefore $\dot{W}(t)$ has a nonnegative projection on the vector $\overrightarrow{0 W(t)}$, see the figure. It means that $W(t)$ does not get closer to the pole 0 , so that $W(t) \notin$ int $M_{0}$.

If now $L_{0}<L$ then, at the moment $t=L_{0}$, one has $W\left(L_{0}\right)=\gamma_{1}\left(L_{0}\right) \in$ int $M_{0}$ which contradicts what is said above.

\section{BIBLIOGRAPHY}

1. A. D. Aleksandrov and V. V. Strel'cov, Isoperimetric problem and estimates of the length of a curve on a surface, Proc. Steklov Inst. Math. 76 (1965), 81-99.

2. B. V. Dekster, Estimates of the length of a curve, J. Differential Geometry 12 (1977), 101-117. 
3. __ Upper estimates of the length of a curve in a Riemannian manifold with boundary, J. Differential Geometry 14 (1979).

4. D. Gromoll and W. Meyer, On complete open manifolds of pasitive curoature, Ann. of Math. (2) 90 (1969), 75-90.

5. Ju. G. Rešetnyak, Bound for the length of a rectifiable curve in n-dimensional space, Sibirsk. Mat. $\check{Z}$. 2 (2) (1961), 261-265. (Russian)

Department of Mathematics, University of Toronto, Toronto M5S 1a7, Ontario, Canada Current address: 406 South 29th Street, South Bend, Indiana 46615 